\begin{tabular}{|c|l|}
\hline Title & A particle field Hamiltonian in relativistic quantum electrodynamics \\
\hline Author(s) & A rai, A sao \\
\hline Citation & Journal of Mathematical Physics, 30(2), 512-520 \\
\hline https://doi.org/10.1063/1.533341 \\
\hline Issue Date & $2000-07$ \\
\hline Doc URL & http://hdl.handle.net/2115/13673 \\
\hline Rights & Copyright $\odot 2000$ A merican Institute of Physics \\
\hline Type & article \\
\hline File Information & jmp41-7.pdf \\
\hline
\end{tabular}

Instructions for use 


\title{
A particle-field Hamiltonian in relativistic quantum electrodynamics
}

\author{
Asao Arai ${ }^{\mathrm{a})}$ \\ Department of Mathematics, Hokkaido University, Sapporo 060-0810, Japan
}

(Received 8 September 1999; accepted for publication 3 April 2000)

We mathematically analyze a Hamiltonian $H_{\tau}(V, g)$ of a Dirac particle-a relativistic charged particle with spin 1/2 - minimally coupled to the quantized radiation field, acting in the Hilbert space $\mathcal{F}:=\left[\oplus^{4} L^{2}\left(\mathbf{R}^{3}\right)\right] \otimes \mathcal{F}_{\text {rad }}$, where $\mathcal{F}_{\text {rad }}$ is the Fock space of the quantized radiation field in the Coulomb gauge, $V$ is an external potential in which the Dirac particle moves, $g$ is a photon-momentum cutoff function in the interaction between the Dirac particle and the quantized radiation field, and $\tau \in \mathbf{R}$ is a deformation parameter connecting the Hamiltonian with the "dipole approximation"' $(\tau=0)$ and the original Hamiltonian $(\tau=1)$. We first discuss the self-adjointness problem of $H_{\tau}(V, g)$. Then we consider $H_{\tau}:=H_{\tau}(0, g)$, the Hamiltonian without the external potential. It is shown that, under a general condition on $g$, the closure of $H_{\tau}$ is unitarily equivalent to a direct integral $\int_{\mathbf{R}^{3}}^{\oplus} \overline{H_{\tau}(\mathbf{p})} d \mathbf{p}$ with a fiber Hamiltonian $H_{\tau}(\mathbf{p})$ acting in the four direct sum $\oplus^{4} \mathcal{F}_{\text {rad }}$ of $\mathcal{F}_{\text {rad }}$, physically the polaron Hamiltonian of the Dirac particle with total momentum $\mathbf{p} \in \mathbf{R}^{3}$. (C) 2000 American Institute of Physics. [S0022-2488(00)05507-9]

\section{INTRODUCTION AND MAIN RESULTS}

In this work we initiate mathematical studies on a quantum system of a Dirac particle-a relativistic charged particle with spin 1/2 - coupled to the quantized radiation field. There may be some models for this quantum system. But, in this article, we investigate the standard model for the quantum system whose Hamiltonian is given by the sum of the Dirac operator with the minimal coupling to the quantized radiation field and the free Hamiltonian of the quantized radiation field. An approximate version of this model was discussed by Bloch and Nordsieck ${ }^{1}$ in view of the infrared problem of quantum electrodynamics. The Hamiltonian they treated is the one obtained by replacing the anticommuting matrices contained in the Dirac operator by $c$-number constants and is much easier to analyze than the original one.

Discussions using informal perturbation methods ${ }^{2}$ suggest that the model may have a physical meaning in a range of quantum electrodynamic phenomena such as the Lamb shift of a hydrogenlike atom and the Compton scattering of the electron where the effects of the quantized radiation field play essential roles. Besides this point, we think that mathematical analysis of the model is interesting also in its own right, because the Hamiltonian of the model belongs to a new class of Hamiltonians on a Hilbert space of Fock type. Moreover the model may be regarded as a model for a quantum mechanical system unstable under the influence of the quantized radiation field. To our best knowledge, no mathematically rigorous analysis has been made on the model so far. $^{3}$

\section{A. Description of the model}

For a Hilbert space $\mathcal{H}$, we denote its inner product and norm by $(\cdot, \cdot)_{\mathcal{H}}($ complex linear in the second variable) and $\|\cdot\|_{\mathcal{H}}$ respectively, but, if there is no danger of confusion, then we often

${ }^{a)}$ Electronic mail: arai@math.sci.hokudai.ac.jp 
omit the subscript $\mathcal{H}$ of them. For a linear operator $T$ on $\mathcal{H}$, we denote its domain by $D(T)$ and by $\sigma(T)$ the spectrum of $T$. For two objects $\mathbf{a}=\left(a_{1}, a_{2}, a_{3}\right)$ and $\mathbf{b}=\left(b_{1}, b_{2}, b_{3}\right)$ such that products $a_{j} b_{j}(j=1,2,3)$ and their sum can be defined, we set $\mathbf{a} \cdot \mathbf{b}:=\sum_{j=1}^{3} a_{j} b_{j}$.

The free Dirac particle of mass $m \geqslant 0$ is described by the free Dirac operator

$$
H_{D}:=\boldsymbol{\alpha} \cdot(-i \boldsymbol{\nabla})+m \beta
$$

acting in the Hilbert space

$$
\mathcal{H}_{D}:=\oplus^{4} L^{2}\left(\mathbf{R}^{3}\right)
$$

with domain $D\left(H_{D}\right):=\oplus^{4} H^{1}\left(\mathbf{R}^{3}\right)\left[H^{1}\left(\mathbf{R}^{3}\right)\right.$ is the Sobolev space of order 1$]$, where $\alpha_{j}(j$ $=1,2,3)$ and $\beta$ are $4 \times 4$ Hermitian matrices satisfying

$$
\begin{gathered}
\left\{\alpha_{j}, \alpha_{k}\right\}=2 \delta_{j k}, \quad j, k=1,2,3, \\
\left\{\alpha_{j}, \beta\right\}=0, \quad \beta^{2}=1, \quad j=1,2,3,
\end{gathered}
$$

$\{A, B\}:=A B+B A$, and $\nabla:=\left(D_{1}, D_{2}, D_{3}\right), D_{j}$ being the generalized partial differential operator in the variable $x_{j}\left[\mathbf{x}=\left(x_{1}, x_{2}, x_{3}\right) \in \mathbf{R}^{3}\right]$. It is well known that $H_{D}$ is self-adjoint and essentially self-adjoint on $\oplus^{4} C_{0}^{\infty}\left(\mathbf{R}^{3} \backslash\{0\}\right)$ (Ref. 4, p.11, Theorem 1.1). Moreover, the spectrum $\sigma\left(H_{D}\right)$ of $H_{D}$ is purely absolutely continuous and

$$
\sigma\left(H_{D}\right)=(-\infty,-m] \cup[m, \infty) .
$$

As for the radiation field, we use the Coulomb gauge in quantizing it. In general, given a Hilbert space $\mathcal{H}$, we have the symmetric (Boson) Fock space

$$
\mathcal{F}_{\mathrm{s}}(\mathcal{H}):=\oplus_{n=0}^{\infty}\left(\otimes_{\mathrm{s}}^{n} \mathcal{H}\right)
$$

over $\mathcal{H}$, where $\otimes_{\mathrm{s}}^{n} \mathcal{H}$ denotes the $n$-fold symmetric tensor product Hilbert space of $\mathcal{H}$ with convention $\otimes_{\mathrm{s}}^{0} \mathcal{H}:=\mathbf{C}$. For basic facts on the theory of the Boson-Fock space, we refer the reader to Ref. 5, Sec. X.7.

The Hilbert space of one-photon states in momentum representation is given by

$$
\mathcal{H}_{\mathrm{ph}}:=L^{2}\left(\mathbf{R}^{3}\right) \oplus L^{2}\left(\mathbf{R}^{3}\right),
$$

where $\mathbf{R}^{3}:=\left\{\mathbf{k}=\left(k_{1}, k_{2}, k_{3}\right) \mid k_{j} \in \mathbf{R}, \quad j=1,2,3\right\}$ physically means the momentum space of photons. The Boson-Fock space

$$
\mathcal{F}_{\text {rad }}:=\mathcal{F}_{\mathrm{s}}\left(\mathcal{H}_{\mathrm{ph}}\right)
$$

over $\mathcal{H}_{\mathrm{ph}}$ serves as a Hilbert space for the quantized radiation field in the Coulomb gauge.

We take a nonnegative Borel measurable function $\omega$ on $\mathbf{R}^{3}$ to denote physically the one free photon energy. We assume that, for almost everywhere (a.e.) $\mathbf{k} \in \mathbf{R}^{3}$ with respect to the Lebesgue measure on $\mathbf{R}^{3}, 0<\omega(\mathbf{k})<\infty$. Then the function $\omega$ defines uniquely a multiplication operator on $\mathcal{H}_{\mathrm{ph}}$ which is nonnegative, self-adjoint and injective. We denote it by the same symbol $\omega$ also. The free Hamiltonian of the quantized radiation field is then defined by

$$
H_{\mathrm{rad}}:=d \Gamma(\omega),
$$

the second quantization of $\omega$. The operator $H_{\text {rad }}$ is a non-negative self-adjoint operator.

Remark 1.1: Usually $\omega$ is taken to be of the form

$$
\omega_{\text {phys }}(\mathbf{k}):=|\mathbf{k}|, \quad \mathbf{k} \in \mathbf{R}^{3},
$$

but, in this paper, for mathematical generality, we do not restrict ourselves to this case. 
We denote by $a(F)\left(F \in \mathcal{H}_{\mathrm{ph}}\right)$ the annihilation operator with test vector $F$ on $\mathcal{F}_{\text {rad }}$. By definition, $a(F)$ is a densely defined closed linear operator and antilinear in $F$. The Segal field operator

$$
\Phi_{\mathrm{s}}(F):=\frac{\overline{a(F)+a(F)^{*}}}{\sqrt{2}}
$$

is self-adjoint, where, for a closable operator $T, \bar{T}$ denotes its closure.

There exist $\mathbf{R}^{3}$-valued continuous functions $\mathbf{e}^{(r)}, r=1,2$, on the nonsimply connected space

$$
\mathbf{M}_{0}:=\mathbf{R}^{3} \backslash\left\{\left(0,0, k_{3}\right) \mid k_{3} \in \mathbf{R}\right\}
$$

such that, for all $\mathbf{k} \in \mathbf{M}_{0}$,

$$
\mathbf{e}^{(r)}(\mathbf{k}) \cdot \mathbf{e}^{(s)}(\mathbf{k})=\delta_{r s}, \quad \mathbf{e}^{(r)} \cdot \mathbf{k}=0, \quad r, s=1,2 .
$$

We set $\mathbf{e}^{(r)}\left(0,0, k_{3}\right):=\mathbf{0}$ for all $k_{3} \in \mathbf{R}$. These vector-valued functions $\mathbf{e}^{(r)}$ are called the polarization vectors of one photon.

Let $g \in L^{2}\left(\mathbf{R}^{3}\right)$. Then, for each $\mathbf{x} \in \mathbf{R}^{3}$ and $j=1,2,3$, we can define an element $g_{j}^{\mathbf{x}}$ of $\mathcal{H}_{\mathrm{ph}}$ by

$$
g_{j}^{\mathbf{x}}(\mathbf{k}):=\left(g(\mathbf{k}) e_{j}^{(1)}(\mathbf{k}) e^{-i \mathbf{k} \cdot \mathbf{x}}, g(\mathbf{k}) e_{j}^{(2)}(\mathbf{k}) e^{-i \mathbf{k} \cdot \mathbf{x}}\right) \in \mathbf{C}^{2} .
$$

Then the quantized radiation field $\mathbf{A}^{g}(\mathbf{x}):=\left(A_{1}^{g}(\mathbf{x}), A_{2}^{g}(\mathbf{x}), A_{3}^{g}(\mathbf{x})\right)$ with momentum cutoff function $g$ is defined by

$$
A_{j}^{g}(\mathbf{x}):=\Phi_{S}\left(g_{j}^{\mathbf{x}}\right), \quad j=1,2,3 .
$$

Remark 1.2: The case $g=1 / \sqrt{(2 \pi)^{3} \omega}$ corresponds to the case without momentum cutoff. We now move to the Hilbert space

$$
\mathcal{F}:=\mathcal{H}_{D} \otimes \mathcal{F}_{\text {rad }}
$$

of state vectors for the coupled system of the Dirac particle and the quantized radiation field. This Hilbert space can be identified as

$$
\mathcal{F}=L^{2}\left(\mathbf{R}^{3} ; \oplus^{4} \mathcal{F}_{\text {rad }}\right)=\int_{\mathbf{R}^{3}}^{\oplus} \oplus^{4} \mathcal{F}_{\text {rad }} d \mathbf{x}
$$

the Hilbert space of $\oplus^{4} \mathcal{F}_{\text {rad }}$-valued Lebesgue square integrable functions on $\mathbf{R}^{3}$ [the constant fiber direct integral with base space $\left(\mathbf{R}^{3}, d x\right)$ and fibre $\oplus^{4} \mathcal{F}_{\text {rad }}$ (Ref. 6, Sec. XIII.6)]. We freely use this identification.

Let $\tau \in \mathbf{R}$ be a constant. Since the mapping $\mathbf{x} \rightarrow g_{j}^{\tau \mathbf{x}}$ from $\mathbf{R}^{3}$ to $\mathcal{H}_{\mathrm{ph}}$ is strongly continuous, we can define a decomposable operator

$$
A_{j}^{g, \tau}:=\int_{\mathbf{R}^{3}}^{\oplus} A_{j}^{g}(\tau \mathbf{x}) d \mathbf{x}
$$

acting in $\mathcal{F}$ which is self-adjoint (Ref. 6, Theorem XIII.85).

We denote by $q \in \mathbf{R} \backslash\{0\}$ the charge of the Dirac particle. We consider the situation where the Dirac particle is in an external potential described by a $4 \times 4$ Hermitian matrix-valued Borel measurable function $V=\left(V_{a b}\right)_{a, b=1, \ldots, 4}$. Then the Hamiltonian of the Dirac particle is given by

$$
H_{D}(V):=H_{D}+V \text {. }
$$


The minimal interaction between the Dirac particle and the quantized radiation field with momentum cutoff $g$ is given by

$$
H_{I, \tau}(g):=-q \boldsymbol{\alpha} \cdot \mathbf{A}^{g, \tau} .
$$

Thus the total Hamiltonian of the coupled system is defined by

$$
H_{\tau}(V, g):=H_{D}(V)+H_{\mathrm{rad}}+H_{I, \tau}(g) .
$$

This is the main object of the mathematical analysis in the present paper.

Remark 1.3: The original Hamiltonian of the model is $H_{1}(V, g)$ (the case $\left.\tau=1\right)$. On the other hand, $H_{0}(V, g)$ (the case $\left.\tau=0\right)$ is the Hamiltonian with the "dipole approximation." Hence $\tau$ is regarded as a deformation parameter connecting the original Hamiltonian and the dipoleapproximated one. Note that the dipole-approximated Hamiltonian $H_{0}(V, g)$ gives a kind of generalization of the standard spin-boson model. The analysis of $H_{0}(V, g)$ may be harder than that of the standard spin-boson model.

Remark 1.4: For a class of $V$, the essential spectrum $\sigma_{\text {ess }}\left(H_{D}(V)\right)$ of $H_{D}(V)$ coincides with that of $H_{D}$ :

$$
\sigma_{\text {ess }}\left(H_{D}(V)\right)=(-\infty,-m] \cup[m, \infty),
$$

so that the discrete spectrum $\sigma_{d}\left(H_{D}(V)\right)$ of $H_{D}(V)$ is a subset of the interval $(-m, m)$ if $m$ is positive (Ref. 4, p. 116, Theorem 4.7). Suppose that (1.21) holds with $\sigma_{d}\left(H_{D}(V)\right)=\left\{E_{n}\right\}_{n=1}^{N}$ $\left(N<\infty\right.$ or $N$ is countably infinite) and that $\left\{\omega(\mathbf{k}) \mid \mathbf{k} \in \mathbf{R}^{3}\right\}=[\nu, \infty)$ with a constant $\nu \geqslant 0$. Then we have

$$
\sigma_{\text {ess }}\left(H_{D}(V)+H_{\text {rad }}\right)=\mathbf{R}
$$

and each $E_{n}$ is an eigenvalue of $H_{D}(V)+H_{\text {rad }}$ embedded in its continuous spectrum. Hence the spectral analysis of $H_{\tau}(V, g)$ includes a perturbation problem of embedded eigenvalues. We consider this problem in a subsequent paper.

Basic hypotheses to analyze $H_{\tau}(V, g)$ are as follows.

Hypothesis (H.1): $g, g / \sqrt{\omega} \in L^{2}\left(\mathbf{R}^{3}\right)$.

Hypothesis (H.2): For all $a, b=1, \ldots, 4, V_{a b}$ is in the set

$$
L^{2}\left(\mathbf{R}^{3}\right)_{\text {loc }}:=\left\{f: \mathbf{R}^{3} \rightarrow \mathbf{C} \text {; Borel measurable }\left.\left|\int_{|\mathbf{x}| \leqslant R}\right| f(\mathbf{x})\right|^{2} d \mathbf{x}<\infty \text { for all } R>0\right\} .
$$

\section{B. The symmetricity and the numerical range of $H_{\tau}(V, g)$}

According to a basic axiom of quantum mechanics that a quantum mechanical observable be represented by a self-adjoint operator on the Hilbert space of state vectors, we first have to examine self-adjointness of the Hamiltonian $H_{\tau}(V, g)$.

For a linear operator $T$ on a Hilbert space $\mathcal{H}$, its numerical range is defined by

$$
\Theta(T):=\left\{(u, T u)_{\mathcal{H}} \mid u \in D(T),\|u\|_{\mathcal{H}}=1\right\}
$$

(Ref. 7, Chap. V, Sec. 3-2).

The following theorem is concerned with symmetricity of $H_{\tau}(V, g)$ and its numerical range.

Proposition 1.1: Let $\tau \in \mathbf{R}$. Assume (H.1) and (H.2). Then $H_{\tau}(V, g)$ is a symmetric operator with $D\left(H_{\tau}(V, g)\right)=D\left(H_{D}\right) \cap D(V) \cap D\left(H_{\mathrm{rad}}\right)$. Moreover

$$
\Theta\left(H_{D}(V)\right) \subset \Theta\left(H_{\tau}(V, g)\right) .
$$

Remark 1.5: The relation (1.23) shows that, if $H_{D}(V)$ is not semibounded (i.e., neither bounded from below nor above), then so is $H_{\tau}(V, g)$. It is well known that, for a wide class of $V$, 
$H_{D}(V)$ is not semibounded (Ref. 4, Chap. 4, Sec. 4.3). Hence, for such a potential $V$, (1.23) implies that $H_{\tau}(V, g)$ is not semibounded. In particular, in the case of the Coulomb potential

$$
V(\mathbf{x})=V_{C}(\mathbf{x}):=-\frac{Z}{|\mathbf{x}|}(Z>0: \text { a constant }),
$$

which is a physically important case, one can show that, under (H.1), $H_{\tau}\left(V_{C}, g\right)$ is not semibounded for all $\tau \in \mathbf{R}$.

Remark 1.6: By the preceding remark, the model may be unphysical in view of stability of matter. From this point of view, we may consider a modified version of the model: Let $E_{D}$ be the spectral measure of $H_{D}$ and $\Lambda_{+}:=E_{D}([0, \infty))$, the projection of $\mathcal{H}_{D}$ onto the non-negative spectral subspace of the free Dirac operator $H_{D}$. Then the operator

$$
H_{\tau}^{\mathrm{BR}}(V, g):=\Lambda_{+} H_{\tau}(V, g) \Lambda_{+}
$$

may be a Hamiltonian for a quantum system of a Dirac particle interacting with the quantized radiation field. This operator is an extended version of the Brown-Ravenhall Hamiltonian ${ }^{8}$ $\Lambda_{+} H_{D}(V) \Lambda_{+}$. As for certain aspects (e.g., self-adjointness, boundedness from below), the operator $H_{\tau}^{\mathrm{BR}}(V, g)$ is more tractable than $H_{\tau}(V, g)$. The model discussed in Ref. 3 is in fact the one described by $H_{\tau}^{\mathrm{BR}}(V, g)$. The method presented below can be applied to the Hamiltonian $H_{\tau}^{\mathrm{BR}}(V, g)$ too and results similar to those on $H_{\tau}(V, g)$ can be established.

\section{Existence of a self-adjoint extension of $H_{\tau}(V, g)$}

We denote by $C_{D}$ the complex conjugation on $\mathcal{H}_{D}:\left(C_{D} f\right)(\mathbf{x}):=\overline{f(\mathbf{x})}, f \in \mathcal{H}_{D}, \mathbf{x} \in \mathbf{R}^{3}$. By Pauli's lemma (Ref. 4, pp. 14 and 74), there exists a $4 \times 4$ unitary matrix $U_{C}$ such that

$$
\begin{gathered}
U_{C}^{2}=I, \quad U_{C} C_{D}=C_{D} U_{C}, \\
U_{C}^{-1} \alpha_{j} U_{C}=\bar{\alpha}_{j}, \quad j=1,2,3, \quad U_{C}^{-1} \beta U_{C}=-\bar{\beta},
\end{gathered}
$$

where, for a matrix $M, \bar{M}$ denotes its complex conjugate.

The following theorem guarantees the existence of a self-adjoint extension of $H_{\tau}(V, g)$.

Theorem 1.2: Let $\tau \in \mathbf{R}$. Assume (H.1) and (H.2). Suppose that $g$ is real-valued and that

$$
U_{C}^{-1} V(\mathbf{x}) U_{C}=\overline{V(-\mathbf{x})}
$$

for a.e. $\mathbf{x}$. Then $H_{\tau}(V, g)$ has a self-adjoint extension.

Remark 1.7: It is obvious that, if $V$ is a real-valued scalar potential in $L^{2}\left(\mathbf{R}^{3}\right)_{\text {loc }}$ with property $V(\mathbf{x})=V(-\mathbf{x})$ a.e. $\mathbf{x} \in \mathbf{R}^{3}$, then it satisfies (1.27). In particular, the Coulomb potential $V=V_{C}$ (Remark 1.5), which is in $L^{2}\left(\mathbf{R}^{3}\right)_{\text {loc }}$, satisfies (1.27).

Condition (1.27) has a physical meaning. Let $P$ be the parity transformation (or the space inversion) on $\mathcal{H}_{D}$ :

$$
(P f)(\mathbf{x}):=f(-\mathbf{x}), \quad f \in \mathcal{H}_{D},
$$

which is unitary. Then the operator

$$
T_{D}:=P U_{C} C_{D}
$$

on $\mathcal{H}_{D}$ is antiunitary. It is easy to see that (1.27) is equivalent to that

$$
T_{D} V \subset V T_{D}
$$


i.e., $V$ commutes with $T_{D}$. The operator $U_{C} C_{D}$ is called the charge conjugation. Hence (1.27) means the invariance of $V$ under the charge conjugation and the parity transformation (the $\mathrm{CP}$ invariance). Thus the self-adjoint extension of $H_{\tau}(V, g)$ in Theorem 1.2 is related to the CP invariance of the external potential $V$. In this sense Theorem 1.2 has an interest.

\section{Essential self-adjointness}

The next problem to be considered is the uniqueness of self-adjoint extension of $H_{\tau}(V, g)$, i.e., essential self-adjointness of it.

We define

$$
\Delta:=\sum_{j=1}^{3} D_{j}^{2}
$$

the Laplacian acting in $\mathcal{H}_{D}$.

Let $\Omega_{0}:=\{1,0,0,0, \ldots\}$ be the Fock vacuum in $\mathcal{F}_{\text {rad }}$. For a subspace $\mathcal{D}$ of $\mathcal{H}_{\text {ph }}$, we define $\mathcal{F}_{\text {rad }}^{\text {in }}(\mathcal{D}) \subset \mathcal{F}_{\text {rad }}$ to be the subspace algebraically spanned by $\Omega_{0}$ and all the vectors of the form

$$
a\left(F_{1}\right) * \cdots a\left(F_{n}\right) * \Omega_{0}, \quad n \geqslant 1, F_{j} \in \mathcal{D}, j=1, \ldots, n .
$$

If $\mathcal{D}$ is dense in $\mathcal{H}_{\text {ph }}$, then $\mathcal{F}_{\text {rad }}^{\text {in }}(\mathcal{D})$ is dense in $\mathcal{F}_{\text {rad }}$.

Theorem 1.3: Let $\tau \in \mathbf{R}$. Assume (H.1), (H.2) and that

$$
\omega g, \quad|\mathbf{k}| g, \quad \frac{|\mathbf{k}| g}{\sqrt{\omega}} \in L^{2}\left(\mathbf{R}^{3}\right) .
$$

Moreover, assume the following (V.1) and (V.2):

(V.1) $V$ is $-\Delta$ bounded.

(V.2) For each $j=1,2,3$ and $a, b=1, \ldots, 4$, the distribution $D_{j} V_{a b}$ is in $L^{2}\left(\mathbf{R}^{3}\right)_{\mathrm{loc}}$ and there exists a constant $c>0$ such that, for all $f \in \oplus^{4} C_{0}^{\infty}\left(\mathbf{R}^{3}\right)$,

$$
\left\|\left(D_{j} V\right) f\right\| \leqslant c\left\|(-\Delta+1)^{1 / 2} f\right\|, \quad j=1,2,3 .
$$

Let $\mathcal{D} \subset \mathcal{H}_{\mathrm{ph}}$ be a core of the self-adjoint operator $\omega$. Then $H_{\tau}(V, g)$ is essentially self-adjoint on $\left[\oplus^{4} C_{0}^{\infty}\left(\mathbf{R}^{3}\right)\right] \otimes_{\text {alg }} \mathcal{F}_{\text {rad }}^{\text {in }}(\mathcal{D})\left(\otimes_{\text {alg }}\right.$ means algebraic tensor product $)$ and its closure is essentially selfadjoint on every core of $-\Delta+H_{\mathrm{rad}}$.

Remark 1.8: Unfortunately Theorem 1.3 does not cover the Coulomb potential case $V$ $=V_{C}$.

As a corollary to Theorem 1.3, we have the following.

Theorem 1.4: Let $\tau \in \mathbf{R}$. Assume (H.1), (H.2), and (1.32). Suppose that $V$ is bounded. Let $\mathcal{D}$ be as in Theorem 1.3. Then $H_{\tau}(V, g)$ is essentially self-adjoint on $\left[\oplus^{4} C_{0}^{\infty}\left(\mathbf{R}^{3}\right)\right] \otimes_{\mathrm{alg}} \mathcal{F}_{\mathrm{rad}}^{\mathrm{in}}(\mathcal{D})$ and its closure is essentially self-adjoint on every core of $\overline{H_{\tau}(0, g)}$.

\section{E. Direct integral decomposition in the case $V=0$} $V$ :

The final topic in this paper is concerned with the Hamiltonian without the external potential

$$
H_{\tau}:=H_{\tau}(0, g)=H_{D}+H_{\mathrm{rad}}+H_{I, \tau}(g) .
$$

This is a Hamiltonian of a relativistic polaron with spin 1/2. We show, as in the case of nonrelativistic polarons ${ }^{9-14}$ or a spinless relativistic polaron, ${ }^{15}$ that $H_{\tau}$ has a natural direct integral decomposition corresponding to a "deformed" translation invariance.

The momentum operator $\mathbf{P}^{\mathrm{rad}}:=\left(P_{1}^{\mathrm{rad}}, P_{2}^{\mathrm{rad}}, P_{3}^{\mathrm{rad}}\right)$ of the quantized radiation field is defined by 


$$
P_{j}^{\mathrm{rad}}:=d \Gamma\left(k_{j}\right),
$$

the second quantization of the multiplication operator $k_{j}$ on $\mathcal{H}_{\mathrm{ph}}$. For each $\tau \in \mathbf{R}$, we define

$$
L(\tau):=\overline{H_{\mathrm{rad}}-\tau \boldsymbol{\alpha} \cdot \mathbf{P}^{\mathrm{rad}}}
$$

acting in the Hilbert space $\mathbf{C}^{4} \otimes \mathcal{F}_{\text {rad }}=\oplus^{4} \mathcal{F}_{\text {rad }}$. Let

$$
H_{I}:=-q \sum_{j=1}^{3} \alpha_{j} A_{j}^{g}(\mathbf{0})=-q \sum_{j=1}^{3} \alpha_{j} \Phi_{S}\left(g_{j}\right)
$$

with

$$
g_{j}(\mathbf{k}):=g_{j}^{\mathbf{0}}(\mathbf{k})=\left(g(\mathbf{k}) e_{j}^{(1)}(\mathbf{k}), g(\mathbf{k}) e_{j}^{(2)}(\mathbf{k})\right) \in \mathbf{C}^{2}
$$

and, for each $\mathbf{p} \in \mathbf{R}^{3}$,

$$
h_{D}(\mathbf{p}):=\boldsymbol{\alpha} \cdot \mathbf{p}+m \beta
$$

In terms of these operators, we define

$$
H_{\tau}(\mathbf{p}):=h_{D}(\mathbf{p})+L(\tau)+H_{I}
$$

acting in $\oplus^{4} \mathcal{F}_{\text {rad }}$.

We introduce a subspace of $\mathcal{F}_{\text {rad }}$ :

$$
\mathcal{F}_{\text {rad }, 0}^{\infty}:=\mathcal{F}_{\text {rad }}^{\text {fin }}\left(C_{0}^{\infty}\left(\mathbf{R}^{3}\right) \oplus C_{0}^{\infty}\left(\mathbf{R}^{3}\right)\right) .
$$

Theorem 1.5: Let $\tau \in \mathbf{R}$. Assume (H.1) and (1.32). Suppose that $\omega \in L^{2}\left(\mathbf{R}^{3}\right)_{\text {loc }}$ Then, for all $\mathbf{p} \in \mathbf{R}^{3}, H_{\tau}(\mathbf{p})$ is essentially self-adjoint on $\oplus^{4} \mathcal{F}_{\mathrm{rad}, 0}^{\infty}$.

Remark 1.9: The operator $H_{\tau}(\mathbf{p})$ may be regarded as a Hamiltonian of a four component spin interacting with a Bose field. In this sense it is an extended version of the standard spin-boson model where the spin is two component. But note that there is a big difference in that $H_{\tau}(\mathbf{p})$ contains a singular term $-\tau \boldsymbol{\alpha} \cdot \mathbf{P}^{\text {rad }}$, which makes the analysis of $H_{\tau}(\mathbf{p})$ more difficult.

Remark 1.10: Let

$$
\omega_{D}(\mathbf{p}):=\sqrt{\mathbf{p}^{2}+m^{2}}
$$

the energy of the free Dirac particle with momentum p. It is well known (or easy to see) that

$$
\sigma\left(h_{D}(\mathbf{p})\right)=\sigma_{d}\left(h_{D}(\mathbf{p})\right)=\left\{ \pm \omega_{D}(\mathbf{p})\right\}
$$

the multiplicity of each eigenvalue being two. Suppose that $\left\{\omega(\mathbf{k})-|\tau||\mathbf{k}| \mid \mathbf{k} \in \mathbf{R}^{3}\right\}=\left[M_{\tau}, \infty\right)$ with some constant $M_{\tau} \geqslant 0$. Then $\sigma_{\text {ess }}\left(h_{D}(\mathbf{p})+L(\tau)\right)=\left[-\omega_{D}(\mathbf{p})+M_{\tau}, \infty\right)$. Hence, if $2 \omega_{D}(\mathbf{p})$ $\geqslant M_{\tau}$, then the eigenvalue $\omega_{D}(\mathbf{p})$ of $h_{D}(\mathbf{p})+L(\tau)$ is embedded in its continuous spectrum. Thus $H_{\tau}(\mathbf{p})$ gives rise to a preturbation problem of embedded (degenerate) eigenvalues. This problem concerns the instability of the Dirac particle with a positive energy under the influence of the quantized radiation field. We will discuss this aspect in a separate paper.

We say that a set $\left\{T_{j}\right\}_{j=1}^{n}$ of self-adjoint operators on a Hilbert space is strongly commuting if the spectral measures of $T_{i}$ and $T_{j}$ commute for all $i, j=1, \ldots, n, i \neq j$.

It is easy to see that $\left\{P_{j}^{\text {rad }}\right\}_{j=1}^{3}$ is strongly commuting. Hence it follows from the three variable functional calculus that, for all $\mathbf{x} \in \mathbf{R}^{3}$, the operator

$$
Q(\mathbf{x}):=\overline{\sum_{j=1}^{3} x_{j} P_{j}^{\mathrm{rad}}}
$$


acting in $\mathcal{F}_{\text {rad }}$ is self-adjoint. Since the mapping: $\mathbf{x} \rightarrow e^{i \tau Q(\mathbf{x})}$ is strongly continuous, we can define a decomposable operator

$$
W_{\tau}:=\int_{\mathbf{R}^{3}}^{\oplus} e^{i \tau Q(\mathbf{x})} d \mathbf{x}
$$

on $\mathcal{F}=\int_{\mathbf{R}^{3}}^{\oplus} \oplus^{4} \mathcal{F}_{\text {rad }} d \mathbf{x}$. It follows that $W_{\tau}$ is unitary.

The Fourier transform on $\mathcal{H}_{D}=\oplus^{4} L^{2}\left(\mathbf{R}^{3}\right)$ can be naturally extended to a unitary operator on $\mathcal{F}$ by

$$
\left(U_{F} \Psi\right)(\mathbf{p}):=\frac{1}{\sqrt{(2 \pi)^{3}}} \int_{\mathbf{R}^{3}} e^{-i \mathbf{p} \cdot \mathbf{x}} \Psi(\mathbf{x}) d \mathbf{x}, \quad \text { a.e. } \mathbf{p} \in \mathbf{R}^{3}, \Psi \in \mathcal{F} .
$$

We define a unitary operator on $\mathcal{F}$ by

$$
U_{\tau}:=U_{F} W_{\tau}
$$

Then we have a direct integral decomposition

$$
U_{\tau} \mathcal{F}=\int_{\mathbf{R}^{3}}^{\oplus} \oplus^{4} \mathcal{F}_{\text {rad }} d \mathbf{p} .
$$

Theorem 1.6: Under the same assumption as in Theorem 1.5, $H_{\tau}$ is essentially self-adjoint and

$$
U_{\tau} \overline{H_{\tau}} U_{\tau}^{-1}=\int_{\mathbf{R}^{3}}^{\oplus} \overline{H_{\tau}(\mathbf{p})} d \mathbf{p} .
$$

Remark 1.11: In Ref. 16, spectral aspects of $H_{\tau}(\mathbf{p})$ (properties of the ground state energy, existence of the ground state, location of the essential spectrum) and $H_{\tau}$ are discussed in the case where (i) $\mu_{\tau}(\mathbf{k}):=\omega(\mathbf{k})-|\tau||\mathbf{k}|>0$ for a.e. $\mathbf{k} \in \mathbf{R}^{3}$ and (ii) $g, g / \sqrt{\mu_{\tau}} \in L^{2}\left(\mathbf{R}^{3}\right)$. But this case does not cover the physical case $\omega(\mathbf{k})=|\mathbf{k}|$.

\section{PROOF OF PROPOSITION 1.1}

For $f \in L^{2}\left(\mathbf{R}^{3}\right)$ and $j=1,2,3$, we define $f_{j} \in \mathcal{H}_{\mathrm{ph}}$ by

$$
f_{j}:=\left(f e_{j}^{(1)}, f e_{j}^{(2)}\right) \in \mathcal{H}_{\mathrm{ph}} .
$$

Using the fact that, for all $\mathbf{k} \in \mathbf{M}_{0}$,

$$
\sum_{r=1}^{2} e_{j}^{(r)}(\mathbf{k}) e_{l}^{(r)}(\mathbf{k})=\delta_{j l}-\frac{k_{j} k_{l}}{|\mathbf{k}|^{2}}, \quad j, l=1,2,3,
$$

we have

$$
\left\|f_{j}\right\|^{2}=\int_{\mathbf{R}^{3}}|f(\mathbf{k})|^{2}\left(1-\frac{k_{j}^{2}}{|\mathbf{k}|^{2}}\right) d \mathbf{k} .
$$

We set

$$
\||| f \mid\|:=\sum_{j=1}^{3}\left\|f_{j}\right\| .
$$

Lemma 2.1: Assume (H.1). Then $D\left(H_{\mathrm{rad}}^{1 / 2}\right) \subset D\left(H_{I, \tau}(g)\right)$ and, for all $\Psi \in D\left(H_{\mathrm{rad}}^{1 / 2}\right)$ and $\varepsilon>0$, 


$$
\left\|H_{I, \tau}(g) \Psi\right\| \leqslant \sqrt{2(1+\varepsilon)}|q|\left\|\frac { g } { \sqrt { \omega } } \left|\| \| H _ { \mathrm { rad } } ^ { 1 / 2 } \Psi \left\|+\frac{|q|}{\sqrt{2}} \sqrt{1+\frac{1}{\varepsilon}}|\|g|\|\mid\| \Psi \| .\right.\right.\right.
$$

Proof: It is well known that, for all $F \in \mathcal{H}_{\mathrm{ph}}$ and $\psi \in D\left(H_{\text {rad }}^{1 / 2}\right), \psi$ is in $D(a(F)) \cap D\left(a(F)^{*}\right)$ and

$$
\|a(F) \psi\| \leqslant\left\|\frac{F}{\sqrt{\omega}}\right\|\left\|H_{\mathrm{rad}}^{1 / 2} \psi\right\|, \quad\left\|a(F)^{*} \psi\right\| \leqslant\left\|\frac{F}{\sqrt{\omega}}\right\|\left\|H_{\mathrm{rad}}^{1 / 2} \psi\right\|+\|F\|\|\psi\| .
$$

Hence, $D\left(H_{\mathrm{rad}}^{1 / 2}\right) \subset D\left(\Phi_{S}(F)\right)$ and

$$
\left\|\Phi_{S}(F) \psi\right\| \leqslant \sqrt{2}\left\|\frac{F}{\sqrt{\omega}}\right\|\left\|H_{\mathrm{rad}}^{1 / 2} \psi\right\|+\frac{1}{\sqrt{2}}\|F\|\|\psi\| .
$$

By this estimate, we have for all $\psi \in D\left(H_{\mathrm{rad}}^{1 / 2}\right)$ and $\mathbf{x} \in \mathbf{R}^{3}$

$$
\left\|A_{j}^{g}(\mathbf{x}) \psi\right\|_{\mathcal{F}_{\text {rad }}} \leqslant \sqrt{2}\left\|\frac{g_{j}}{\sqrt{\omega}}\right\|\left\|H_{\text {rad }}^{1 / 2} \psi\right\|_{\mathcal{F}_{\text {rad }}}+\frac{1}{\sqrt{2}}\left\|g_{j}\right\|\|\psi\|_{\mathcal{F}_{\text {rad }}} .
$$

Let $\Psi$ be in the domain $D\left(H_{\mathrm{rad}}^{1 / 2}\right)$ as a subspace of $\mathcal{F}$. Then, by (2.8) and the elementary inequality

$$
(a+b)^{2} \leqslant(1+\varepsilon) a^{2}+\left(1+\frac{1}{\varepsilon}\right) b^{2}, \quad a, b \geqslant 0, \varepsilon>0,
$$

we have for a.e. $\mathbf{x}$

$$
\left\|A_{j}^{g}(\tau \mathbf{x}) \Psi(\mathbf{x})\right\|_{\mathcal{F}_{\text {rad }}}^{2} \leqslant 2(1+\varepsilon)\left\|\frac{g_{j}}{\sqrt{\omega}}\right\|^{2}\left\|H_{\mathrm{rad}}^{1 / 2} \Psi(\mathbf{x})\right\|_{\mathcal{F}_{\text {rad }}}^{2}+\frac{1}{2}\left(1+\frac{1}{\varepsilon}\right)\left\|g_{j}\right\|^{2}\|\Psi(\mathbf{x})\|_{\mathcal{F}_{\text {rad }}}^{2} .
$$

Integrating the both sides with respect to $\mathbf{x}$, we see that $\Psi \in D\left(A_{j}^{g, \tau}\right)$ with

$$
\left\|A_{j}^{g, \tau} \Psi\right\| \leqslant \sqrt{2(1+\varepsilon)}\left\|\frac{g_{j}}{\sqrt{\omega}}\right\|\left\|H_{\mathrm{rad}}^{1 / 2} \Psi\right\|+\frac{1}{\sqrt{2}} \sqrt{1+\frac{1}{\varepsilon}}\left\|g_{j}\right\|\|\Psi\| .
$$

Thus $\Psi \in \cap_{j=1}^{3} D\left(A_{j}^{g, \tau}\right)=D\left(H_{I, \tau}(g)\right)$. By using the fact $\left\|\alpha_{j}\right\|=1$ and (2.10), we obtain (2.5).

Proof of Proposition 1.1: Lemma 2.1 implies that $D\left(H_{\tau}(V, g)=D\left(H_{D}\right) \cap D(V) \cap D\left(H_{\text {rad }}\right)\right.$. By (H.2), we have $\oplus^{4} C_{0}^{\infty}\left(\mathbf{R}^{3}\right) \subset D(V)$. Hence $D\left(H_{\tau}(V, g)\right)$ is dense. It is easy to see that $\left(\Psi, H_{\tau}(V, g) \Phi\right)=\left(H_{\tau}(V, g) \Psi, \Phi\right)$ for all $\Psi, \Phi \in D\left(H_{\tau}(V, g)\right)$. Thus $H_{\tau}(V, g)$ is a symmetric operator.

Let $\lambda \in \Theta\left(H_{D}(V)\right)$. Then $\lambda=\left(f, H_{D}(V) f\right)$ for some $f \in D\left(H_{D}(V)\right)$ with $\|f\|=1$. Let $\Psi_{f}$ $:=f \Omega_{0}$. Then $\Psi_{f} \in D\left(H_{\tau}(V, g)\right),\left\|\Psi_{f}\right\|=1$. Using the fact that $a(F) \Omega_{0}=0, F \in \mathcal{H}_{\mathrm{ph}}$ and $H_{\mathrm{rad}} \Omega_{0}$ $=0$, we have

$$
\left(\Psi_{f}, H_{\tau}(V, g) \Psi_{f}\right)=\left(f, H_{D}(V) f\right)=\lambda .
$$

Hence $\lambda \in \Theta\left(H_{\tau}(V, g)\right)$. Thus (1.23) follows.

\section{PROOF OF THEOREMS 1.2-1.4}

A mapping $C$ on a Hilbert space is called a conjugation if it is antilinear, norm-preserving and $C^{2}=I$ (identity). 
Lemma 3.1: Let $C$ be a conjugation on a Hilbert space $\mathcal{H}$ and $S$ be a linear operator on $\mathcal{H}$ such that $C S \subset S C$. Then $C S=S C$.

Proof: We need only to show that $D(S C) \subset D(S)$. Let $\psi \in D(S C)$. Then $C \psi \in D(S)$. Hence, by the assumption, $C(C \psi) \in D(S)$ and $S C(C \psi)=C S(C \psi)$, which means that $\psi \in D(S)$ and $S \psi=C S C \psi$. Thus the assertion follows.

Note that the mapping $T_{D}$ defined by (1.29) is a conjugation. Using (1.26) and Lemma 3.1, one can easily prove the following lemma.

Lemma 3.2: For $j=1,2,3$,

$$
T_{D} \alpha_{j}=\alpha_{j} T_{D}, \quad T_{D} D_{j}=-D_{j} T_{D}
$$

In particular,

$$
T_{D} \boldsymbol{\alpha} \cdot(-i \boldsymbol{\nabla})=\boldsymbol{\alpha} \cdot(-i \boldsymbol{\nabla}) T_{D}
$$

Let $C_{\mathrm{ph}}$ be the complex conjugation on $\mathcal{H}_{\mathrm{ph}}$ :

$$
C_{\mathrm{ph}}\left(F_{1}, F_{2}\right):=\left(\overline{F_{1}}, \overline{F_{2}}\right) \in \mathcal{H}_{\mathrm{ph}}, \quad F_{r} \in L^{2}\left(\mathbf{R}^{3}\right), \quad r=1,2,
$$

and $j_{\mathbf{C}}$ be the complex conjugation on $\mathbf{C}$. Then

$$
J_{\mathrm{rad}}:=j_{\mathbf{C}} \oplus\left(\oplus_{n=1}^{\infty} \otimes{ }^{n} C_{\mathrm{ph}}\right)
$$

is a conjugation on $\mathcal{F}_{\text {rad }}$.

Lemma 3.3: We have

$$
J_{\mathrm{rad}} H_{\mathrm{rad}}=H_{\mathrm{rad}} J_{\mathrm{rad}}
$$

and, for all $F \in \mathcal{H}_{\mathrm{ph}}$,

$$
J_{\mathrm{rad}} \Phi_{S}\left(C_{\mathrm{ph}} F\right)=\Phi_{S}(F) J_{\mathrm{rad}}
$$

Proof: Relation (3.5) follows from the reality of $\omega$. As for (3.6), we first show that $\Phi_{S}(F) J_{\mathrm{rad}}=J_{\mathrm{rad}} \Phi_{S}\left(C_{\mathrm{ph}} F\right)$ on the subspace of finite particle vectors

$$
\mathcal{F}_{\text {rad }, 0}:=\left\{\psi=\left\{\psi^{(n)}\right\}_{n=0}^{\infty} \in \mathcal{F}_{\text {rad }} \mid \psi^{(n)}=0 \text { for all but finitely many } n \text { 's }\right\} .
$$

Then, by a limiting argument using the fact that $\mathcal{F}_{\text {rad,0 }}$ is a core of $\Phi_{S}(F)$, we see that $J_{\mathrm{rad}} \Phi_{S}\left(C_{\mathrm{ph}} F\right) \subset \Phi_{S}(F) J_{\mathrm{rad}}$. By this fact and Lemma 3.1, we obtain (3.6).

Proof of Theorem 1.2: The operator $J:=T_{D} \otimes J_{\text {rad }}$ acting in $\mathcal{F}$ is a conjugation. By Lemmas 3.2 and 3.3 and the present assumption, we have $J\left(H_{\tau}(V, g)-m \beta\right)=\left(H_{\tau}(V, g)-m \beta\right) J$. Hence, by von Neumann's theorem (Ref. 5, Theorem X.3), $H_{\tau}(V, g)-m \beta$ has a self-adjoint extension $H_{\tau}^{\prime}(V, g)$. Since $m \beta$ is bounded, $H_{\tau}^{\prime}(V, g)+m \beta$ is self-adjoint by the Kato-Rellich theorem (Ref. 5, Theorem X.12). It is obvious that $H_{\tau}^{\prime}(V, g)+m \beta$ is a self-adjoint extension of $H_{\tau}(V, g)$.

Proof of Theorem 1.3: The method of proof is to apply Nelson's commutator theorem (Ref. 5, Theorem X.37). We take as the comparison operator the self-adjoint operator

$$
K_{0}:=-\Delta+H_{\mathrm{rad}}+1 \geqslant 1 \text {. }
$$

We recall a useful identity: Let $T_{j}, j=1,2,3$, be linear operators on a Hilbert space $\mathcal{H}$ such that $T_{j} T_{\ell}=T_{\ell} T_{j}$ on $D\left(T_{j} T_{\ell}\right) \cap D\left(T_{\ell} T_{j}\right), j, \ell=1,2,3$, and set $\mathbf{T}:=\left(T_{1}, T_{2}, T_{3}\right)$. Then, by using (1.3), one can easily show that

$$
(\boldsymbol{\alpha} \cdot \mathbf{T})^{2}=\mathbf{T}^{2} \text { on } \cap_{j, \ell=1}^{3}\left[D\left(T_{j} T_{\ell}\right) \cap D\left(T_{\ell} T_{j}\right)\right],
$$

where $\alpha_{j} T_{j}(j=1,2,3)$ is considered as an operator on $\oplus^{4} \mathcal{H}$. 
Let $f \in D(-\Delta)$. Then, by (3.8),

$$
\|\boldsymbol{\alpha} \cdot(-i \boldsymbol{\nabla}) f\|^{2}=(f,-\Delta f) \leqslant\|f\|\|-\Delta f\| .
$$

By this inequality and (V.1), we can show that

$$
\left\|H_{D}(V) f\right\| \leqslant c_{1}\|(-\Delta+1) f\|,
$$

where $c_{1}>0$ is a constant. By the elementary inequality

$$
a b \leqslant \varepsilon a^{2}+\frac{1}{4 \varepsilon} b^{2},
$$

holding for all $a>0, b>0, \varepsilon>0$, we have for all $\Psi \in D\left(H_{\text {rad }}\right)$

$$
a\left\|H_{\mathrm{rad}}^{1 / 2} \Psi\right\| \leqslant a\|\Psi\|^{1 / 2}\left\|H_{\mathrm{rad}} \Psi\right\|^{1 / 2} \leqslant \varepsilon\left\|H_{\mathrm{rad}} \Psi\right\|+\frac{a^{2}}{4 \varepsilon}\|\Psi\| .
$$

Hence, by Lemma 2.1, for all $\varepsilon>0$, there exists a constant $b_{\varepsilon} \geqslant 0$ such that

$$
\left\|H_{I, \tau}(g) \Psi\right\| \leqslant \varepsilon\left\|H_{\mathrm{rad}} \Psi\right\|+b_{\varepsilon}\|\Psi\|, \quad \Psi \in D\left(H_{\mathrm{rad}}\right) .
$$

Estimates (3.9) and (3.10) imply that

$$
\left\|H_{\tau}(V, g) \Phi\right\| \leqslant c_{2}\left\|K_{0} \Phi\right\|, \quad \Phi \in D\left(K_{0}\right),
$$

where $c_{2}>0$ is a constant.

Let $\Psi \in\left[\oplus^{4} C_{0}^{\infty}\left(\mathbf{R}^{3}\right)\right] \otimes_{\text {alg }} \mathcal{F}_{\text {rad }}^{\text {in }}(\mathcal{D})$. Then

$$
\left(H_{D}(V) \Psi, K_{0} \Psi\right)-\left(K_{0} \Psi, H_{D}(V) \Psi\right)=2 i \sum_{j=1}^{3} \Im\left(\left(D_{j} V\right) \Psi, D_{j} \Psi\right) .
$$

Hence, using (V.2) and the fact that

$$
\left\|D_{j} \Psi\right\| \leqslant\left\|(-\Delta)^{1 / 2} \Psi\right\| \leqslant\left\|K_{0}^{1 / 2} \Psi\right\|,
$$

we can show that

$$
\left|\left(H_{D}(V) \Psi, K_{0} \Psi\right)-\left(K_{0} \Psi, H_{D}(V) \Psi\right)\right| \leqslant c_{3}\left\|K_{0}^{1 / 2} \Psi\right\|^{2},
$$

where $c_{3}>0$ is a constant. We have

$$
\left(H_{I, \tau}(g) \Psi, \Delta \Psi\right)-\left(\Delta \Psi, H_{I, \tau}(g) \Psi\right)=-2 i q \sum_{j, \ell=1}^{3} \Im\left(D, \Psi, \alpha_{j} B_{\ell j} \Psi\right),
$$

where $B_{/ j}:=\int_{\mathbf{R}^{3}}^{\oplus} \Phi_{S}\left(\partial g_{j}^{\tau \mathbf{x}} / \partial x_{\ell}\right) d \mathbf{x}$. By Lemma 2.1 with $g$ replaced by $-i k_{/} g$, we can estimate $\left\|B_{\ell j} \Psi\right\|$. Hecne we obtain

$$
\left|\left(H_{I, \tau}(g) \Psi,-\Delta \Psi\right)-\left(-\Delta \Psi, H_{I, \tau}(g) \Psi\right)\right| \leqslant c_{4}\left\|K_{0}^{1 / 2} \Psi\right\|^{2},
$$

where $c_{4}>0$ is a constant. It is easy to see that

$$
\left[H_{I, \tau}(g), H_{\mathrm{rad}}\right] \Psi=q \sum_{j=1}^{3} \alpha_{j} B_{j} \Psi,
$$


where $B_{j}:=\int_{\mathbf{R}^{3}}^{\oplus} \Phi_{S}\left(i \omega g_{j}^{\tau \mathbf{x}}\right) d \mathbf{x}$. By Lemma 2.1 with $g$ replaced by $i \omega g$, we can estimate $\left\|B_{j} \Psi\right\|$ to obtain

$$
\left|\left(H_{I, \tau}(g) \Psi, H_{\mathrm{rad}} \Psi\right)-\left(H_{\mathrm{rad}} \Psi, H_{I, \tau}(g) \Psi\right)\right| \leqslant c_{5}\left\|K_{0}^{1 / 2} \Psi\right\|^{2},
$$

where $c_{5}>0$ is a constant. Since $\left(H_{\mathrm{rad}} \Psi, K_{0} \Psi\right)-\left(K_{0} \Psi, H_{\mathrm{rad}} \Psi\right)=0$, it follows that

$$
\left|\left(\left[H_{\mathrm{rad}}+H_{I, \tau}(g)\right] \Psi, K_{0} \Psi\right)-\left(K_{0} \Psi,\left[H_{\mathrm{rad}}+H_{I, \tau}(g)\right] \Psi\right)\right| \leqslant\left(c_{4}+c_{5}\right)\left\|K_{0}^{1 / 2} \Psi\right\|^{2} .
$$

The subspace $\left[\oplus^{4} C_{0}^{\infty}\left(\mathbf{R}^{3}\right)\right] \otimes_{\text {alg }} \mathcal{F}_{\text {rad }}^{\text {fin }}(\mathcal{D})$ is a core of $K_{0}$. Thus, by (3.11), (3.12), and (3.14), we can apply Nelson's commutator theorem to obtain the desired result.

Proof of Theorem 1.4: We write $H_{\tau}(V, g)=H_{\tau}(0, g)+V$. By Theorem 1.3, $H_{\tau}(0, g)$ is essentially self-adjoint on $\left[\oplus^{4} C_{0}^{\infty}\left(\mathbf{R}^{3}\right)\right] \otimes_{\text {alg }} \mathcal{F}_{\text {rad }}^{\text {fin }}(\mathcal{D})$. Since $V$ is bounded, we can apply the KatoRellich theorem to obtain the desired result.

\section{PROOF OF THEOREMS 1.5 AND 1.6}

We define a deformed total momentum operator $\mathbf{P}(\tau):=\left(P_{1}(\tau), P_{2}(\tau), P_{3}(\tau)\right)$ with parameter $\tau \in \mathbf{R}:$

$$
P_{j}(\tau):=\overline{-i D_{j}+\tau P_{j}^{\mathrm{rad}}}
$$

on $\mathcal{F}(j=1,2,3)$. Each $P_{j}(\tau)$ is self-adjoint and its spectrum is purely absolutely continuous with

$$
\sigma\left(P_{j}(\tau)\right)=\mathbf{R} .
$$

Physically $P_{j}(\tau)$ is interpreted as the generator of a unitary representation of a (deformed) translation to the $j$ th direction. It is not difficult to $\operatorname{see}^{16}$ that, for all $t \in \mathbf{R}$,

$$
e^{i t P_{j}(\tau)} H_{\tau} \subset H_{\tau} e^{i t P_{j}(\tau)} .
$$

This shows a deformed translation invariance of $H_{\tau}$.

We can show that, for $j=1,2,3$,

$$
U_{\tau} P_{j}(\tau) U_{\tau}^{-1}=\int_{\mathbf{R}^{3}}^{\oplus} p_{j} d \mathbf{p} .
$$

Thus the Hilbert space $U_{\tau} \mathcal{F}$ carries a spectral representation of $\mathbf{P}(\tau)$ and the index parameter $\mathbf{p}$ in the decomposition (1.47) physically means an observed value of the deformed total momentum $\mathbf{P}(\tau)$.

Proof of Theorem 1.5: Let $\mathbf{p} \in \mathbf{R}^{3}$ be fixed and $L:=\sum_{j=1}^{3}\left(p_{j}-\tau P_{j}^{\mathrm{rad}}\right)^{2}+H_{\mathrm{rad}}+1$. Then $L$ is self-adjoint, non-negative and reduced by each closed subspace $\oplus^{4}\left(\otimes_{s}^{n} \mathcal{H}_{\mathrm{ph}}\right)$ with its reduced part $L_{n}$ being the multiplication self-adjoint operator by the funciton $\left(\mathbf{p}-\tau \sum_{j=1}^{n} \mathbf{k}_{j}\right)^{2}+\sum_{j=1}^{n} \omega\left(\mathbf{k}_{j}\right)$ +1 . By the present assumption, this function is in $L^{2}\left(\mathbf{R}^{3 n}\right)_{\text {loc }}$. Hence $L_{n}$ is essentially self-adjoint on $\oplus^{4} S_{n}\left(\otimes_{\text {alg }}^{n}\left[C_{0}^{\infty}\left(\mathbf{R}^{3}\right) \oplus C_{0}^{\infty}\left(\mathbf{R}^{3}\right)\right]\right)$, where $S_{n}$ denotes the symmetrizer on $\otimes^{n} \mathcal{H}_{\mathrm{ph}}$. It follows that $L$ is essentially self-adjoint on $\oplus^{4} \mathcal{F}_{\text {rad, } 0}^{\infty}$. Let $\psi \in \oplus^{4} \mathcal{F}_{\text {rad, } 0}^{\infty}$. Then, by (3.8),

$$
\left\|\boldsymbol{\alpha} \cdot\left(\mathbf{p}-\tau \mathbf{P}^{\mathrm{rad}}\right) \psi\right\| \leqslant\left\|L^{1 / 2} \psi\right\| .
$$

In the same way as in Lemma 2.1, we can show that

$$
\left\|H_{I} \psi\right\| \leqslant \sqrt{2}|q|\left\|\frac { g } { \sqrt { \omega } } \left|\|\| H_{\mathrm{rad}}^{1 / 2} \psi\left\|+\frac{|q|}{\sqrt{2}}|||g|||\right\| \psi \| .\right.\right.
$$

By these estimates, we obtain $\left\|H_{\tau}(\mathbf{p}) \psi\right\| \leqslant c_{1}\|L \psi\|$ with a constant $c_{1}>0$. In the same way as in the proof of Theorem 1.3, we can show that 


$$
\left|\left(H_{\tau}(\mathbf{p}) \psi, L \psi\right)-\left(L \psi, H_{\tau}(\mathbf{p}) \psi\right)\right| \leqslant c_{2}\left\|L^{1 / 2} \psi\right\|^{2},
$$

with a constant $c_{2}>0$. Thus we can apply Nelson's commutator theorem to obtain the desired result.

Lemma 4.1: Let $z \in \mathbf{C} \backslash \mathbf{R}$. Then, under the same assumption as in Theorem 1.5, the operatorvalued funciton: $\mathbf{p} \rightarrow\left(\overline{H_{\tau}(\mathbf{p})}-z\right)^{-1}$ on $\mathbf{R}^{3}$ is strongly continuous.

Proof: The set $\mathbf{C} \backslash \mathbf{R}$ is a subset of the resolovent set of $\overline{H_{\tau}(\mathbf{p})}$, since $\overline{H_{\tau}(\mathbf{p})}$ is self-adjoint. Let $\mathbf{p}, \mathbf{p}^{\prime} \in \mathbf{R}^{3}$. Then, for all $\psi \in \oplus^{4} \mathcal{F}_{\mathrm{rad}, 0}^{\infty}$, we have by (3.8) $\left\|H_{\tau}\left(\mathbf{p}^{\prime}\right) \psi-H_{\tau}(\mathbf{p}) \psi\right\|=\left|\mathbf{p}^{\prime}-\mathbf{p}\right|\|\psi\|$ $\rightarrow 0\left(\mathbf{p}^{\prime} \rightarrow \mathbf{p}\right)$. Since $\oplus^{4} \mathcal{F}_{\text {rad, } 0}^{\infty}$ is a common core for the family $\left\{\overline{H_{\tau}(\mathbf{p})}\right\}_{\mathbf{p} \in \mathbf{R}^{3}}$ of self-adjoint operators, it follows from a convergence theorem [Ref. 17, Theorem VIII.25(a)] that $\left(\overline{H_{\tau}\left(\mathbf{p}^{\prime}\right)}-z\right)^{-1}$ $\rightarrow\left(\overline{H_{\tau}(\mathbf{p})}-z\right)^{-1}$ strongly as $\mathbf{p}^{\prime} \rightarrow \mathbf{p}$.

Proof of Theorem 1.6: The essential self-adjointness of $H_{\tau}$ follows from the present assumption and Theorem 1.4. By Theorem 1.5, Lemma 4.1 and a general theorem [Ref. 6, Theorem XIII.85(a)], we have $H_{\tau}^{\prime}:=\int_{\mathbf{R}^{3}}^{\oplus} \overline{H_{\tau}(\mathbf{p})} d \mathbf{p}$ is self-adjoint. On the other hand, by direct computation, we see that $U_{\tau} H_{\tau} U_{\tau}^{-1} \subset H_{\tau}^{\prime}$. The essential self-adjointness of $H_{\tau}$ implies that of $U_{\tau} H_{\tau} U_{\tau}^{-1}$. Thus (1.48) follows.

\section{ACKNOWLEDGMENT}

This work is supported by the Grant-in-Aid No. 11440036 for Scientific Research from the Ministry of Education, Science, Sports and Culture.

${ }^{1}$ F. Bloch and A. Nordsieck, "Notes on the radiation field of the electron," Phys. Rev. 52, 54-59 (1937).

${ }^{2}$ K. Nishijima, Relativistic Quantum Mechanics (Baihu-kan, Tokyo, 1973)(in Japanese).

${ }^{3}$ Compare however, R. T. Prosser, "On the energy spectrum of the hydrogen atom in a photon field. I," J. Math. Phys. 39, 229-277 (1998). In this paper a modified version of the model is considered with relatively much mathematical rigor.

${ }^{4}$ B. Thaller, The Dirac Equation (Springer-Verlag, Berlin, Heidelberg, 1992).

${ }^{5}$ M. Reed and B. Simon, Methods of Modern Mathematical Physics II: Fourier Analysis, Self-Adjointness (Academic, New York, 1975).

${ }^{6}$ M. Reed and B. Simon, Methods of Modern Mathematical Physics IV: Analysis of Operators (Academic, New York, 1978).

${ }^{7}$ T. Kato, Perturbation Theory for Linear Operators (Second Edition, Springer-Verlag, Berlin, Heidelberg, 1976).

${ }^{8}$ G. E. Brown and D. G. Ravenhall, "On the interaction of two electrons," Proc. R. Soc. London, Ser. A 208, 552-559 (1952).

${ }^{9}$ J. T. Cannon, "Quantum field theoretic properties of a model of Nelson," J. Funct. Anal. 8, 101-152 (1971).

${ }^{10}$ L. Gross, "Existence and uniqueness of physical ground states," J. Funct. Anal. 10, 52-109 (1972).

${ }^{11}$ J. Fröhlich, "On the infrared problem in a model of scalar electrons and mass-less, scalar bosons," Ann. Inst. Henri Poincare, Sect. A 19, 1-103 (1973).

${ }^{12}$ J. Fröhlich, "Existence of dressed one electron states in a class of persistent models," Fortschr. Phys. 22, 159-198 (1974).

${ }^{13}$ H. Spohn, "Effective mass of the polaron: a functional integral approach," Ann. Phys. (N.Y.) 175, 278-318 (1988).

${ }^{14}$ H. Sophn, "The polaron at large total momentum," J. Phys. A 21, 1199-1211 (1988).

${ }^{15}$ L. Gross, "The relativistic polaron without cutoffs," Commun. Math. Phys. 31, 25-73 (1973).

${ }^{16}$ A. Arai, "Fundamental properties of the Hamiltonian of a Dirac particle coupled to the quantized radiation field," Hokkaido University Preprint Series \#447, February 1999.

${ }^{17}$ M. Reed and B. Simon, Methods of Modern Mathematical Physics I: Functional Analysis (Academic, New York, 1972). 